\title{
EXTRACTION OF CAUSAL-ASSOCIATION NETWORKS FROM UNSTRUCTURED TEXT DATA
}

\author{
A Thesis \\ presented to \\ the Faculty of California Polytechnic State University \\ San Luis Obispo \\ In Partial Fulfillment \\ of the Requirements for the Degree \\ Master of Science in Computer Science
}

by

Brett N. Bojduj

June 2009 
(C) 2009

Brett Nicholas Bojduj

ALL RIGHTS RESERVED 


\section{COMMITTEE MEMBERSHIP}

TITLE:

AUTHOR:

DATE SUBMITTED:
Extraction of Causal-Association Networks from Unstructured Text Data

Brett N. Bojduj

June 2009

COMmittee ChaiR: Franz J. Kurfess, Ph.D.

COMmitTeE MemBer: Clark S. Turner, Ph.D.

COMmitTeE MEMBER: Jens G. Pohl, Ph.D. 


\begin{abstract}
Extraction of Causal-Association Networks from Unstructured Text Data

by

Brett N. Bojduj
\end{abstract}

Causality is an expression of the interactions between variables in a system. Humans often explicitly express causal relations through natural language, so extracting these relations can provide insight into how a system functions. This thesis presents a system that uses a grammar parser to extract causes and effects from unstructured text through a simple, pre-defined grammar pattern. By filtering out non-causal sentences before the extraction process begins, the presented methodology is able to achieve a precision of $85.91 \%$ and a recall of $73.99 \%$. The polarity of the extracted relations is then classified using a Fisher classifier. The result is a set of directed relations of causes and effects, with polarity as either increasing or decreasing. These relations can then be used to create networks of causes and effects. This "Causal-Association Network" (CAN) can be used to aid decision-making in complex domains such as economics or medicine, that rely upon dynamic interactions between many variables. 


\section{Acknowledgements}

First I would like to thank God for preserving my life thus far, opening up numerous opportunities for me, blessing me with great and loving parents, and giving me the strength and motivation to try to do what is right. I should also thank my parents for putting up with me well enough to let me live at home all these years that I have spent at Cal Poly. Their love and (financial) support have been most appreciated and seeing their dedication to me has helped me to stay motivated when life has been hard.

I would also like to thank Dennis Taylor of CDM Technologies, Inc., for collaborating with me so successfully over the past several years. I have learned a lot about research from his guidance and it has made me a better scientist. Also, thanks to Ben G. Weber of the University of California at Santa Cruz. My collaborations with him have also helped grow me as a scientist and his comments on this thesis were much appreciated.

My committee should also be thanked for reading this long thesis and giving their comments. Special thanks to my advisor, Dr. Franz Kurfess for his great patience and dedication to helping me and his other students. Over the past several years I have learned a lot from Dr. Kurfess and without his help, this thesis would never have happened.

Very special thanks to NEC Corporation and especially to Hideki Kawai, without whom this work would not have been possible. The work presented in this thesis was accomplished at the NEC C\&C Innovation Research Laboratories in Nara, Japan. 


\section{Contents}

List of Tables viii

List of Figures ix

1 Introduction 1

1.1 Importance of Causality . . . . . . . . . . . . . 2

1.2 Defining Causality . . . . . . . . . . . . . . 3

1.3 Motivation ..................... . . 4

1.4 Contributions ......................... 4

1.5 A Running Example ... . . . . . . . . . . . 5

1.6 Outline ........................ 7

2 Related Work 9

2.1 Causal Sufficiency and Spurious Correlations . . . . . . . . . . . . 11

2.2 Inferring Causal Relations from Empirical Data . . . . . . . . . 11

2.2.1 Causal Bayesian Reasoning . . . . . . . . . . . 13

2.3 Extracting Causal Relations from Textual Data . . . . . . . . . 15

2.3.1 Causal Extraction Based on Grammar Patterns . . . . . . 16

2.3.2 Causal Extraction Based on Co-Occurrence . . . . . . . . 19

2.4 Related Work Summary . . . . . . . . . . . . . . 21

3 Causal-Association Networks $\quad 22$

3.1 Governing Assumptions . . . . . . . . . . . . . . 23

3.2 Direction of Causal Relations . . . . . . . . . . . . . . . . . . 24

3.3 Polarity of Causal Relations . . . . . . . . . . . . . . . 24 
3.4 Causal-Association Network Generation . . . . . . . . . . . . . 25

3.4.1 Causal Verbs ................... 26

3.4 .2 Sentence Extraction . . . . . . . . . . . . . 26

3.4 .3 Sentence Filtering . . . . . . . . . . . . . . . . . . . . . . . 28

3.4.4 Tuple Extraction . . . . . . . . . . . . . . . . 32

3.4.5 Polarity Classification ............... . 34

3.4.6 Tuple Scoring . . . . . . . . . . . . . . . . 35

3.4.7 Influence Calculation . . . . . . . . . . . . . . . . 35

3.5 Visualization .................... 36

3.6 Summary Overview of Causal Association Networks . . . . . . . . 38

4 Framework for Extracting Causal-Association Networks from the $\begin{array}{ll}\text { Internet } & 39\end{array}$

4.1 Causal Tuple Extraction from the Internet . . . . . . . . . . . . . 40

4.1.1 Querying for Text Data ............... 42

4.1.2 Sentence Extraction from the Internet . . . . . . . . . . . 43

4.2 New Query Generation . . . . . . . . . . . . . . . . . 44

4.3 Summary of Extracting Causal-Association Networks from the Internet ....................... . . 4 44

5 Verification and Results $\quad 45$

5.1 Metrics......................... . . 45

5.2 Causal and Polarity Classification Results . . . . . . . . . . . 46

5.2.1 Sources of Error . . . . . . . . . . . . . . 47

5.3 Causal Tuple Extraction Results . . . . . . . . . . . . . . . . . . 47

5.3.1 Sources of Error . . . . . . . . . . . . . . . . . . 48

5.4 Summary of Results . . . . . . . . . . . . . . . . 50

6 Conclusions and Future Work $\quad 51$

6.1 Conclusion . . . . . . . . . . . . . . . 52

6.2 Future Work . . . . . . . . . . . . . . . . 52

6.2.1 List of Open Problems . . . . . . . . . . . . . 53

$\begin{array}{ll}\text { Bibliography } & 55\end{array}$ 


\section{List of Tables}

2.1 Table of Features of Selected Related Works . . . . . . . . . . . . 16

3.1 List of Verbs . . . . . . . . . . . . . . . . . . 27

3.2 List of Stop Words . . . . . . . . . . . . . . . . . . . . . . . . . . 29

3.3 List of Increasing Words . . . . . . . . . . . . . . . . . . . 30

3.4 List of Decreasing Words . . . . . . . . . . . . . . . . . . 30

5.1 Causal Tuple Extraction Results . . . . . . . . . . . . . . . . . . 48 


\section{List of Figures}

1.1 Graph of Causal Relations for the Late 2000s American Financial Recession . . . . . . . . . . . . . . . 7

2.1 Causal Bayesian Network . . . . . . . . . . . . . . . . . . . . 14

3.1 Causal Tuple Format . . . . . . . . . . . . . . . . . . . . . . . . 22

3.2 Overview of Causal-Association Network Generation . . . . . . 25

3.3 Parse Tree . . . . . . . . . . . . . . . . . . . . . . . . . . . 32

3.4 Visualization for Financial Domain . . . . . . . . . . . . 37

4.1 Overview of Causal Tuple Extraction . . . . . . . . . . . . . 41

5.1 Visualization for Extracted Medical Abstract Data . . . . . . . 48 


\section{Chapter 1}

\section{Introduction}

To a human, a sentence like "A supertanker is released by Somali pirates after a ransom is dropped onto the ship by parachute, reports say." (from BBC RSS feed, Jan. 9, 2009) clearly implies a causal relation between one event (drop of a ransom) and another (release of a supertanker), although the sentence explicitly only contains the temporal relation "after." Examples such as this are indicative of the ubiquity of causality in quotidian life. In one way or another causality

affects us all, since causality expresses the dynamics of a system. Thus it can be useful to the understanding of a system if the causal relations in the system are known. In order to explicate the causal relations within a domain, humans often express causal relations through natural language text. Therefore, extracting these causal relations can not only provide insight into how a system functions, but also can be used as a measure of the perceptions that people have of a system and/or domain, since natural language explanation of causal relations can often be expressed as opinion. 


\subsection{Importance of Causality}

Lotfi Zadeh, the father of fuzzy logic, once wrote [51] that, "Causality occupies a position of centrality in human cognition." This is a strong statement by any standards, but it is indicative not only of Zadeh's zeal for causality, but also of the importance of causality in human reasoning and decision-making. Causality is important for decision-making across many domains because it is a description of the interactions between variables in a domain. Since people make decisions that change the state of a system, understanding how changes to a system will affect system dynamics is crucial.

Hoover [12] states that "The study of causality in any particular context is the study of the particular connections that permit control of one thing to influence another." Particularly in the domain of economics, causality is a key to being able to explain, predict, and control the outcomes of events. Since economic systems, when viewed as a complex adaptive system may exhibit "rapidly diminishing returns" [33], a causal understanding of an economic system may lead to a person or an organization being able to impose some measure of control on the system to produce desired results.

It is a natural desire for engineers, in particular, to try to change a system to remedy apparent faults. However, oftentimes the causa sine qua non of a fault in a complex system is not readily apparent. In circumstances such as these, an analysis of the dynamics of the system must be performed and the causes of faults in the system must be found. In this way, causes and their effects can be seen as a key to provide insight into how to change a system for the better. Perhaps this is why Democritus proclaimed, "I would rather discover one causal law than be the King of Persia" [28]. 


\subsection{Defining Causality}

Despite the importance of causality in understanding the dynamics between variables in a system, defining it is difficult, at best. Zadeh [51, 52] goes as far as to say that causality is "undefinable," given current paradigms of logic and reasoning. However, just because something is undefinable in a general way that is universally applicable does not mean that it is undefinable if one merely wants to explicate a definition for a specific purpose. Thus causality can often be defined in domain-specific terms, but that definition is unlikely to be applicable across domains. Thus for many cases, an imprecise definition of causality must be used.

Picard $[31]^{1}$ quotes John McCarthy discussing imprecise knowledge:

We can't define Mt. Everest precisely-whether or not a particular rock or piece of ice is or isn't part of it; but it is true, without qualification, that Edmund Hillary and Tenzing Norgay climbed it in 1953. In other words, we can base solid facts and knowledge on structures that are themselves imprecisely defined.

Thus for the purpose of explicating a standard, domain-independent definition to be used throughout this work, we define causality as follows:

A causal relationship exists between two atomic entities, $\alpha$ and $\beta$, if $\alpha$ has an influence upon $\beta$. Direction and strength of the relation is determined by the net influence of one atomic entity on the other. The relationship between $\alpha$ and $\beta$ where $\alpha$ causes $\beta$ will be represented in this work by $\alpha \prec \beta$.

This definition is certainly not perfect, as words such as "influence" are necessarily vague, but it is a definition which is good enough for our purposes here. As Zadeh [51] states, "any attempt to define causality within the conceptual structure of classical logic and probability theory has no chance of success." Since the

\footnotetext{
${ }^{1}$ Page 21.
} 
aim of this work is not to present a new paradigm for logical reasoning, we shall continue, using our definition from above.

\subsection{Motivation}

The main focus of this work is the extraction of a causal-association network (not to be confused with causal associational network (CASNET) models from the medical expert system domain, c.f. [48]) from unstructured text data. This work relies upon causal relations that other people have explicated through human natural language. Relying on mining causal relations from natural language text shifts the problem of causal-association network generation from that of probability-based inference of causes and effects, to that of an unstructuredtext data mining problem. Though we will briefly discuss some previous work on probabilistic-based inference for obtaining causal relations in Section 2.2, this paper will focus mainly on the practical extraction and use of causal relations from the standpoint of an unstructured-text data mining problem. For future work, using a causal-inference approach, such as a Bayesian network or influence diagram would be a good way to bridge the gap between mathematical causal modeling and practical data mining techniques.

\subsection{Contributions}

The main contribution of this work is a simplified method for cause and effect extraction using a grammar parse tree, whereas related work has relied on a large number of grammar patterns or very simple patterns that are unable to extract causal relations from more complex sentences. 
This thesis also makes an argument for the determination of the polarity of causal relations, as a measure of how a cause affects an effect. To determine the polarity of a causal relation, this thesis shows that a Fisher classifier (c.f. Section 3.4.5) can be used.

Additionally, this thesis presents the use of a Fisher classifier as a method for filtering out non-causal sentences so that erroneous causes and effects are not extracted from non-causal sentences. Through empirical tests (c.f. Chapter 5), it is shown that this causal/non-causal sentence filter can boost precision with only small amount of harm to recall, thus increasing the composite F-score.

\subsection{A Running Example}

In a lecture on causality at IJCAI'99, Judea Pearl said, "The subject of my lecture this evening is causality. It is not an easy topic to speak about, but it is a fun topic to speak about. It is not easy because, like religion, sex and intelligence, causality was meant to be practiced, not analyzed" [29]. Thus, since causality is meant to be practiced, the discussion of causal relations and their extraction will be grounded in a concrete example. The example that will be used for this analysis is the late 2000s American financial recession that is, as of this writing, still being played out. The fact that the causal relations in this domain are changing quickly as new information becomes available highlights both the strengths and weaknesses of the causal reasoning approaches presented in this paper. A good analogy for causal relations in this respect is that just as mathematical equations define a system as it is, not as it will be, so it is with causal relations. This is in keeping with the assertion that causal relations describe how variables in a system interact. 
The example of the late 2000s American financial recession that we will use for our work is shown in Figure 1.1. We focus on the domain of economics for our use case because causality in the economic domain is readily understandable, at least in a qualitative sense. In the words of Herbert Simon [45], economics “concerns itself with a particular subset of man's behaviors-those having to do with the production, exchange, and consumption of goods and services." Thus, as an artifact of human behavior, economics should have a broad interest to many people.

In particular, Sah and Stiglitz $[35,36]$ talk about the importance of structure in economic systems. The structure of an economic system greatly affects the strengths and types of causal relations that occur in the system. Thus we will focus on an economic structure that concerns the following variables (the relations of which are shown in Figure 1.1):

- Li's Gaussian Copula

- Sub-prime lending

- Credit Default Swap

- Collateralized Debt Obligation

- Housing Market

- Late 2000s Recession

- Deregulation

- Keynesian Resurgence

- National Debt 


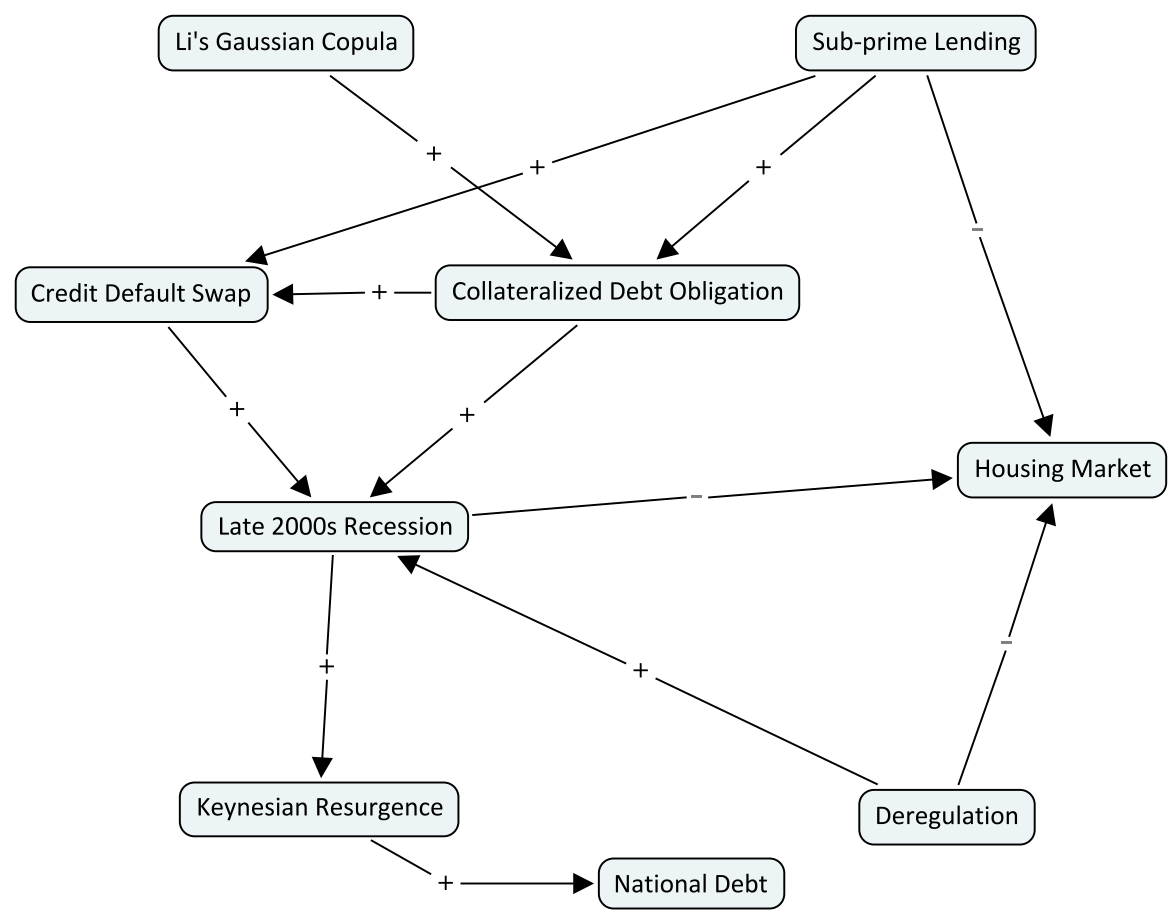

Figure 1.1: Graph of Causal Relations for the Late 2000s American Financial Recession

Though some of the relations between variables in Figure 1.1 may be disputable, the veracity of the relations is not central to this work and thus one should not spend an undue effort to worry about it. The purpose of the relations is merely to provide a semi-realistic example that can be used to clarify causal relation extraction techniques.

Throughout the examples of causation, the notation " $\alpha \prec \beta$ " ( $\alpha$ causally precedes $\beta$ ) shall be used as a concise way to represent a directed causal relation.

\subsection{Outline}

Related work is discussed in Chapter 2. For the discussion of related work, we touch briefly on general interest in causality and theoretical causal inferenc- 
ing before delving into previously applied practical methods of causal relation extraction from unstructured natural language text data. Based on the related work, we introduce the formalism of causal-association networks (CAN) to represent causal-relations extracted from textual data, in Chapter 3. An exemplary system for extracting causal-association networks from unstructured text data on the Internet is describe in Chapter 4 and results of the causal-relation extraction approach is in Chapter 5. Finally, conclusions and possible directions for future work are discussed in Chapter 6. 


\section{Chapter 2}

\section{Related Work}

Interest in causality began very early in recorded history. Democritus was one of the earliest recorded people to attempt to explain how one event causes another [26]. However it was Hume who sparked much of the modern interest in causality [29] by arguing for an empirical basis for causality. Pinker [32], though half in jest, concisely explains Hume's empiricism when he writes:

"For many mornings, then, over a span of years, I have experienced the chime of the PDA followed a number of seconds later by the blare of the clock. And according to a well-known theory of perception of causation associated with the philosopher David Hume, I should think that the chime causes the blare." 1

Though perhaps an overly simplistic example, this illustrates the difficulty in determining causality, since even direct empirical evidence cannot always distinguish between causation and correlation, without the input of knowledge about a domain. Section 2.1 will discuss this problem in further detail. Though believed by many to be flawed in his conclusions, Hume played an important role in shaping commonsense beliefs about the empirical nature of causality that many

\footnotetext{
${ }^{1}$ Page 153.
} 
have today. In particular, Hoover [12] details Hume's interest in causality for determining economic policy.

At around the same time that Hume explored causality, Bayes [1] developed the basis for a mathematical framework for the formal treatment of events and probabilities. With the contemporary availability of significant computational power, today Bayesian methods are used to answer some of the causal questions that Hume explored, namely how causality can be inferred from non-causal data about an event or situation.

The dynamics between variables in a system is often crucial. Brooks [2] states that "Intelligence is determined by the dynamics of interaction with the world." This statement goes along with Simon's [46] assertion that the behavioral complexity of humans is a function of the complexity of the environment they are embedded in (since Simon argues that the internal complexity of humans is low).

Domains, such as economics, where humans try to make decisions to affect a desired change may often contain more causal relations, or conflicting causal relations, than humans can easily manage. This is especially true when these causal relations are described in natural language text, where large quantities of text must be scanned in order to extract the requisite knowledge for decision making. To help with this task, computer-aided causal reasoning and causal relation extraction techniques have been developed.

Related work on causality can be divided into two categories: inferring causal relations from empirical data and extracting causal relations that are already made explicit. 


\subsection{Causal Sufficiency and Spurious Correlations}

A set of variables is typically defined as causally sufficient if all the causes for all the variables of the set are included in the set [41]. In sets that are not causally sufficient, it is difficult to ascertain whether or not two variables are causally related or merely correlated, with a common cause. This work, and much of the related works, does not explicitly consider causal sufficiency, but this is something the reader should keep in mind. One reason this is not a significant issue in this thesis is because the extracted causal relations have already been made explicit by a human through natural language text. Thus it is hoped the human who writes about causal relations in a domain knows enough about the domain so they can tell the difference between causation and correlation before they write about causal relations.

In the 1950's, Simon attempted to tackle the problem of how to determine whether or not a correlation between two variables was either causal or merely a "spurious correlation" [44]. In order to determine causation, exogenous variables must be taken into consideration to see whether or not other variables are causally connected. Simon shows mathematically that if you change the value of the exogenous variables, then based on how the other variables change, you can tell whether or not two variables in the system are causally related.

\subsection{Inferring Causal Relations from Empirical Data}

Much work was done by Simon on defining and deriving causal relations based on causal ordering $[42,43,44]$. Simon's work focused primarily on inferring 
causality based on structure [13]. A notable feature of Simon's theory of causal ordering is that it is atemporal. To determine that one variable causes another, no information about which came first is needed. This is, perhaps, in contrast to a common-sense definition of causality in which causes have to precede events, but it is useful in domains where equations are used to define the dynamics of a system, but the temporal order of the variables in the equations is either not known or not specified. In such a situation, causality has to be derived based on the interactions and not on the temporal ordering and that is what Simon's causal ordering theory accomplishes.

Simon treated causality as a derived entity based on the relations between mathematical variables. In some situations it is not possible to tell whether or not one variable causes another or not. To resolve which variable is an effect, exogenous data is needed to resolve the causal ordering. The reason for this is that there is no inherent ordering algebraically in the mathematical equations of variables that would state that one thing causes another, rather than the other way around.

Iwasaki $[15,16,14]$ worked with Simon to extend the theory of causal ordering to allow for feedback loops, such as those that occur in economic and physical systems.

Whereas Simon's theory of causal ordering focused on deriving causality from non-causal mathematical equations, Lin [22] treated causality as a mathematical primitive unto itself and worked to extend situation calculus [25] for causal reasoning. This extension of situation calculus allows one to formally represent actions and their effects in situation calculus, which enables causal modeling of systems that are described using situation calculus. McCain and Turner [23, 24] worked on causal knowledge representation and uses for causal knowledge. Lang, 
Lin, and Marquis [21] created a formal computational model for McCain and Turner's work.

Despite this extensive work on causality there is still no general definition of causality that will work in every domain and every situation. This is a testament to the difficulty of formally defining a concept that humans intuitively understand via a commonsense definition.

\subsubsection{Causal Bayesian Reasoning}

Since the 1980's Bayesian networks have frequently been used for computing causal relations [28] by using Bayes's theorem to determine the conditional probability of one variable occurring with another. The causal feasibility of this co-occurrence can be calculated using the mathematical techniques of Bayesian networks.

Bayesian networks are directed acyclic graphs (DAGs) [27, 28] where the nodes in the graph are independent from each other if they are $d$-separated [3]. D-separation means that two nodes in a Bayesian DAG are conditionally independent from each other given another, third node. Charniak [3] explains dseparation as the state of not being $d$-connected, where two nodes are d-connected if there is a "causal path" connecting them or if they are somehow correlated.

Bayesian networks can be used for inferring causal relations from statistical data by assigning probabilities to different nodes in the network. Since the network is directed, preceding nodes can be thought of to cause nodes which occur later in the graph. An example of a causal Bayesian network based on the running example in this paper is shown in Figure 2.1. Given probabilities for parent nodes in the network, the probability of a node occurring can be calculated, as shown 


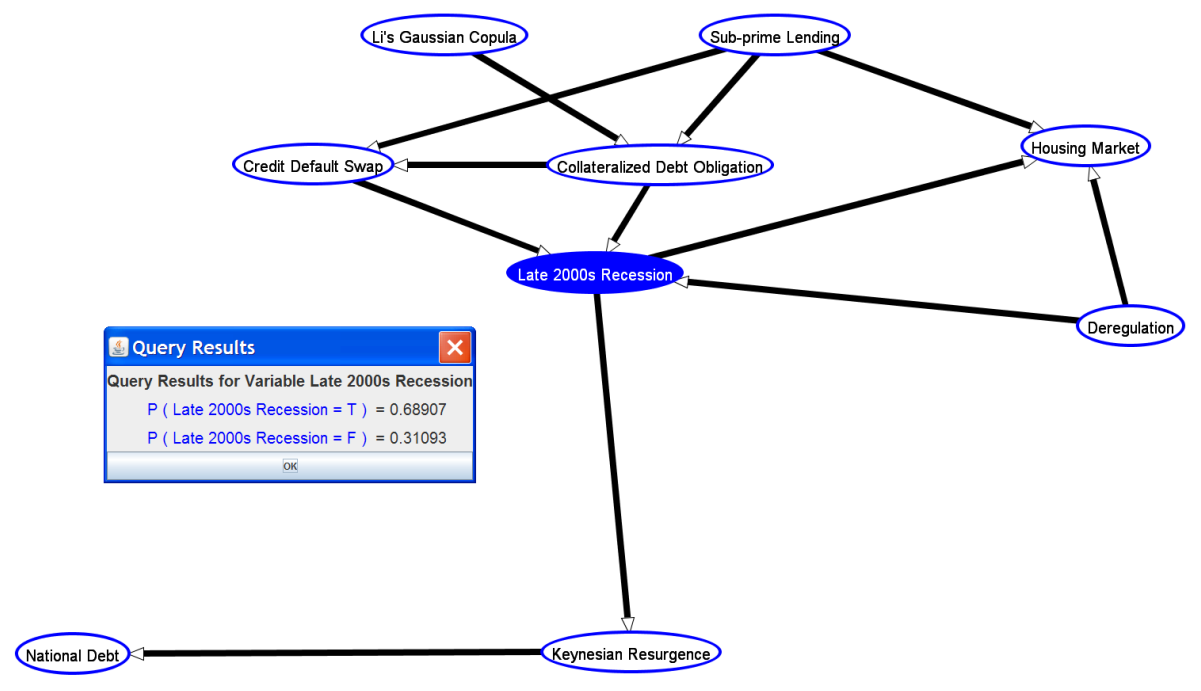

Figure 2.1: Causal Bayesian Network

in the figure. Criticism of this methodology exists [7], however, since there is no fundamental direction of the graph which is explicitly found in the probabilities of the data.

One major advantage of Bayesian networks is that they can be used to model situations probabilistically, which is useful when an incomplete description of a system or an event must be used for reasoning. However, a disadvantage of using Bayesian networks is that often finding exact solutions is an NP hard problem [5, 49], thus restrictions must be placed on problem representation or approximation techniques must be used [3].

As shown in this section, many approaches to causal reasoning take advantage of statistics and mathematical reasoning in order to infer causal relations from empirical data or infer new causal relations based on statistics between known relations. However, mathematical and statical inference is not the only way to deal with causal relations. Humans often express causality in natural language, thus there is a significant field of work which attempts not to infer causal relations, 
but rather to extract those relations which are already explicated in natural language text. Though explicitly explicated causal relations in natural language may be subject to inaccuracies due to people misrepresenting causal knowledge or merely presenting inaccurate opinions about a causal relationship between two things, even extracting these relations can be of use because they are a way to quantitatively measure the perceptions that people may have. Thus extracting causal relations can be a useful endeavor. It is this body of work that is the focus of the next section.

\subsection{Extracting Causal Relations from Textual Data}

Comparing related works dealing with causality is difficult since, not only is causality "undefinable" [51, 52], but there are also no standard data sets that are used to compare one approach to another. Part of the reason for this is the difficulty and expense of creating and hand-annotating data sets for causal extraction tasks. Therefore, each paper typically uses its own dataset in an ad hoc manner and the best the authors can do is hint that their approach is better than others based on results from their own data set. However, as is typical with information extraction papers, many authors compute precision and recall statistics that can be used to give a rough idea of how one paper compares to others in the field.

Causal extraction from text data can be subdivided into two main approaches: extraction based on grammar patterns and extraction based on co-occurrence statistics. A summary of some of the related works that will be discussed is 
shown in Table 2.1.

\begin{tabular}{|c|c|c|c|c|}
\hline & $\begin{array}{c}\text { Khoo } \\
\text { et al., } \\
2000[18]\end{array}$ & $\begin{array}{c}\text { Girju, } \\
2003[9]\end{array}$ & $\begin{array}{c}\text { Sanchez-Graillet } \\
\text { and Poesio, } \\
2004[39]\end{array}$ & $\begin{array}{c}\text { Perrin } \\
\text { et al., } \\
2008[30]\end{array}$ \\
\hline Single Sentence & $\mathrm{X}$ & $\mathrm{X}$ & $\mathrm{X}$ & \\
\hline Multiple Sentences & & & & $\mathrm{X}$ \\
\hline Directed Relation & $\mathrm{X}$ & $\mathrm{X}$ & $\mathrm{X}$ & \\
\hline Precision/Recall & & $77.9 \% / 88.7 \%$ & $60 \% / \mathrm{NA}$ & $60 \% / 80 \%$ \\
\hline Accuracy & $58 \%$ & & & \\
\hline
\end{tabular}

Table 2.1: Table of Features of Selected Related Works

\subsubsection{Causal Extraction Based on Grammar Patterns}

Much work has been done in data mining on extracting causal relations from unstructured text data. Girju [9], Girju and Moldovan [10], Khoo, et al. [18], and Sanchez-Graillet and Poesio [39] used simple grammar patterns for extracting causes and effects. These patterns consisted of causes, a causal verb, and an effect, in order to extract explicitly-stated causal relations. For example, a sentence that matches this pattern is "Deregulation decreases the housing market." The extracted causal relation from this sentence would be "Deregulation $\prec$ housing market."

Khoo, et al. [18] used information obtained from a sentence's parse tree to determine causes and effects based on the grammar structure with the causal verb. Using a medical dataset, they were able to obtain a $58 \%$ accuracy for extracting causes and a $51 \%$ accuracy for extracting effects, where both causes and effects were stated explicitly in the text data and fit their causal patterns. Unfortunately, they do not provide precision and recall statistics for their tests, so it is difficult to compare their work with other works.

To extract causal relations, Girju [9] and Girju and Moldovan [10] used a 
sentence parser which tagged the parts of speech of words in a sentence. From this, noun phrases were extracted from both sides of a causal verb. However, noun phrases that are extracted by this approach are often longer than they need to be. For example, consider the sentence, "Deregulation causes a devalued housing market." Using a sentence parser would give you "deregulation" as the first noun phrase and "devalued housing market" as the second noun phrase. However, if you only wanted "housing market" and not "devalued housing market" to be extracted, then this could cause problems in your system. To get around this, Girju [9] uses WordNet [8] to lookup the different words that make up the noun phrases and then takes the largest group of words that appears as a complete phrase in WordNet. It should be noted that in reality, Girju's approach would not work for the example that we explicated in Figure 1.1, since phrases like "housing market" are not in WordNet.

One significant work that has attempted to link causal inference and causal extraction is that by Sanchez-Graillet and Poesio [39], who attempt to automatically create Bayesian networks from causal relations extracted from unstructured text. Their work is similar to the work by Girju and Moldovan [10], Girju [9], and Khoo, et al. [18], in that their system extracts causal relations based on noun-verb-noun grammar patterns. In addition to naively picking out causes and effects from these verb patterns, Sanchez-Graillet and Poesio also look at conjunctions, disjunctions, and negations within the verb patterns. For example, in the conjunctive sentence, "Li's gaussian copula and collateralized debt obligations caused the credit default swap market to expand," their system would extraction the causal relation "Li's gaussian copula, collateralized debt obligations $\prec$ credit default swap market." Sanchez-Graillet and Poesio's system can also handle disjunctive sentences such as, "Deregulation or sub-prime lending brought down the 
housing market," the causal relations "deregulation $\prec$ housing market" and "subprime lending $\prec$ housing market" are extracted from that sentence. However, for disjunctions the problem of only one of the multiple causes being true makes this handling of disjunctions somewhat questionable. Still, in order to support the extraction of more complex cause and effect relations, a trade-off will often need to be made between the precision of the extraction and the recall.

Sanchez-Graillet and Poesio [39] then take the extracted causal relations and build a Bayesian network from them. The probabilities of the nodes are based on frequency. Though they report a precision of $60 \%$, they do not report any recall value, so it is hard to properly evaluate their results. However, one of the possible reasons for their low precision is that basing the probabilities of the nodes on frequency is not necessarily a good way to go about building a Bayesian network, since there is an almost unprocessable amount of textual data available, such as on the Internet. Therefore, unless you can get a truly random sample of text data, which is exceedingly unlikely, then you cannot know what the frequency of a term or causal relation means.

Higashinaka and Isozaki [11], though focused on answering why-questions in Japanese, were able to apply automatically extracted causal relations to their work. They used abstracted grammar parse-trees in causally-annotated sentences with BACT [19], a tree-based machine-learning method, to extract common grammar patterns that occur in the causal sentences. They then used the extracted grammar patterns to create features used in their generated answers to why-questions.

Higashinaka and Isozaki's [11] work is notable in that it is one of the few works that actually uses the extracted causal relations to perform a task. Most of the works that are focused on extracting causal relations from text data are more 
concerned with the method of extraction for causal relations than on how the causal relations can be used. One could argue that this is out of necessity rather than a lack of focus or creativity, since extracting two terms and saying that they are causally related only says just that, i.e., that they are causally related, rather than how they are causally related. Thus an open area for future work is extracting not just that two terms are causally related, but to also infer how they are related, that is, the polarity of a causal relation. This thesis incorporates polarity, defined in Section 3.3, as part of the causal-association network structure for causal relations, described in Chapter 3.

\subsubsection{Causal Extraction Based on Co-Occurrence}

Rather than relying on grammar patterns, Saito et al. [37] and Perrin et al. [30] used co-occurrence of "statistical terms" to build networks from text data. They defined a "statistical term" to be a measurement of some kind of quantity that is important to a system. For example, in an economic domain, statistical terms might be terms like "consumer interest rate" or "inflation." Whereas Saito et al. [37] focused on single sentences (in Japanese), Perrin et al. [30] did not consider sentences at all and merely focused on distance between statistical terms in text. Thus if Perrin et al.'s method was looking for possible causal relations between "deregulation" and "housing market" and was given the following text:

"Greed promoted deregulation. In turn, this caused the housing market to make a sharp downturn."

then they would extract "deregulation" and "housing market" as causally related. Note that there is no direction of the relation, but merely co-occurrence, thus we cannot say that "deregulation $\prec$ housing market." The main disadvan- 
tage of both Saito et al. [37] and Perrin et al.'s [30] works are the direction of a causal relation cannot be inferred simply based on co-occurrence. Also, their definition of causality as simply the co-occurrence of two terms together in text data is somewhat questionable. However, to be fair, it is in keeping with a "commonsense" definition of causality that two terms occurring often together will most likely affect the causal dynamics of a system. Comparing to Simon's causal ordering theory, however, it seems that exogenous terms are needed to test whether or not two terms are really causally related.

Sakai and Masuyama [38] extracted terms from Japanese-language financial articles. They then considered these terms to be causes affecting business performance based on co-occurrence with a "clue expression," such as “好调 (are good)." Using their method, they achieved a precision of $79.2 \%$ and a recall of $66.1 \%$. An advantage of this view of causality is that finding causes is fairly easy, since the effect, i.e., business performance, is known, so one just has to look for a clue expression and extract phrases that are attached to that as the cause. However, a disadvantage of their approach is that their definition of causality relies upon "clue expression" phrases specific to a domain, such as business performance, in order to perform the extraction. This hurts the flexibility of their system and also requires a priori knowledge as to what clue expressions should be used in order to find certain information. In other words, you need to have an idea of what you are looking for before you start, if you use their method. In some domains, such as business performance for a specific company, this will work. However, if you are trying to understand the complex causal dynamics of a very large system, then their method may not be suitable. 


\subsection{Related Work Summary}

Causal relations are useful to extract since they express the dynamics between variables in a system. Two main approaches to causality are mathematical inference techniques, such as Bayesian networks, and data mining techniques that extract causal relations from natural language text.

Current work on extracting causal relations from text is fragmented and ad hoc. Part of the reason for this is the lack of a standardized data set that everyone uses and the lack of a standardized definition of causality (the possibility of which is questionable). However, we can still make progress in this field by improving the techniques of causal extraction to deal with features like multiple-sentences, direction, and polarity (a measure of "how" one thing affects another, formally discussed in Section 3.3) of relations. Focusing on improving support for these features is a good topic for future work.

Furthermore, any future work dealing with causal relation extraction should also compute and publish precision and recall statistics, as well as make available data sets that were used in the work. Perhaps if enough authors do this, the field will be able to mature at a greater rate. 


\section{Chapter 3}

\section{Causal-Association Networks}

In order to improve the field of causal relation extraction (c.f., Chapter 2), this thesis proposes the use of causal-association networks as a structure for causal relations extracted from unstructured text data ${ }^{1}$. A causal-association network (CAN) is a network of causal tuples with the format shown in Figure 3.1.

<cause phrase, verb phrase, effect phrase, polarity>

Figure 3.1: Causal Tuple Format

Notable features of the causal relations in a causal-association network are the direction of the relations (that is, $\alpha$ causes $\beta$, and not the other way around) and polarity of the relations. Classifying the polarity of causal relations is an important contribution, since polarity describes the how of the relation, e.g., how A affects B. Section 3.3 discusses polarity in detail.

\footnotetext{
${ }^{1}$ Similar networks have long been used in the expert systems community, e.g., in causal associational networks CASNET [20]. Though similar in name, the causal-association networks $(\mathrm{CAN})$ presented in this thesis are not the same thing.
} 


\subsection{Governing Assumptions}

Extracting causal relations from unstructured text data is currently a large field, mainly due to the growth of textual data available on the Internet. However, implicit in the extraction of causal relations from text data are the following assumptions:

Meaningful descriptions of causality occur in textual data Research suggests that the causal structure of descriptions of events is a useful aid in the understanding of those descriptions [47]. This would point out the utility of causal relations depicted in natural language text.

Causal relations are often explicitly stated in the same sentence In their study of causality, Khoo et al. [18] found that in medical texts, $93 \%$ of causal relations are both explicit and in the same sentence. Furthermore, Iwasaki and Simon [15] point out that "in informal descriptions of realworld phenomena, statements of the form, 'A causes B,' are exceedingly common." This would lend credence to the assumption that at the very least, mining textual data for explicit causal relations is a worthy pursuit.

Group intelligence If many people describe reality as a certain way, then perhaps that description is relevant.

Taken as a whole, these governing assumptions provide a simplified view of causality and reality that can more easily be worked with. This provides a clear and distinct basis on which we can discuss work that has attempted to extract explicit causal relations from textual data. 


\subsection{Direction of Causal Relations}

A notable feature of causality is the asymmetric nature of its relations [34]. Knowing that A causes B does not tell you anything about B causing A (and in fact may decrease that prospect). Therefore the direction of causal relations is important and thus an extraction method that can determine the direction of the extracted causal relation must be used. In this work the direction is determined based on output from a grammar parser, where the grammar extraction pattern is constructed so the cause will almost always be on the left side of a causal verb and the effect on the right side.

\subsection{Polarity of Causal Relations}

As a common-sense definition of polarity, it can be considered to be the "how" a cause affects an effect in a causal relation. That is, does the cause increase or decrease the effect, or is there not a clear change (i.e., is the affect upon the effect neutral)?

Since the utility of polarity may be unclear when discussed in an abstract way, we will now clarify increasing, decreasing, and neutral polarities through the use of concrete examples.

Example of Increasing Polarity A Keynesian resurgence increases the national debt. Extracted tuple: < "Keynesian resurgence," "increases," "national debt," positive>

Example of Decreasing Polarity Risky subprime loans decrease the value of the housing market. Extracted tuple: < "subprime loans," "decline," "value 
housing market," negative>

Example of Neutral Polarity Subprime lending affects the housing market.

Extracted tuple: <"Subprime lending," "affects," "housing market," neutral>

As shown in the previous examples, polarity is a measure of how a cause interacts with an effect to change the value of the effect in some way.

\subsection{Causal-Association Network Generation}

The generation of the causal-association network relies upon calculating the net influences (Section 3.4.7) between tuples of causes, effects, causal verbs, and polarity, extracted from sentential data. In order to extract this set of tuples of causal relations, defined in Figure 3.1, the process shown in Figure 3.2 is performed.

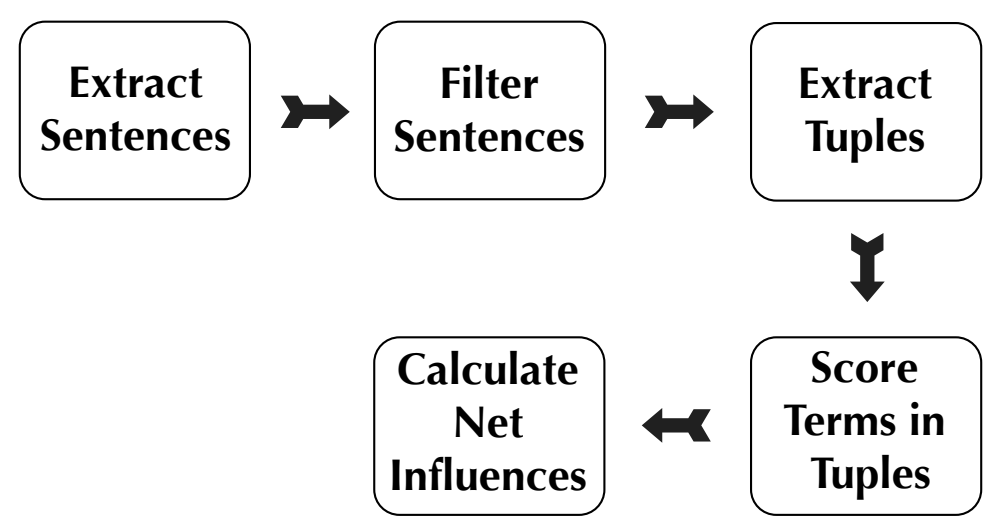

Figure 3.2: Overview of Causal-Association Network Generation

The causal extraction method is based on extracted noun and adjectival

phrases associated with a causal verb. Though the noun phrases used belong to an a priori-undefined, open set of any nouns and adjectives, the list of causal 
verbs is a closed set that is not added to once the system runs. Therefore, the set of causal verbs used to extract causal relations must be defined.

\subsubsection{Causal Verbs}

Causal verbs are taken as singular, plural, and present participle forms of the verb "cause" and all troponyms of the verb "cause," as defined in WordNet [8]. In addition to the verbs extracted from WordNet, the following verbs were added to our list of causal verbs: kill, dictate, impose, provide, affect, result, increase, promote, reduce, complicate, decrease, and play. A complete list of causal verbs is shown in Table 3.1 .

\subsubsection{Sentence Extraction}

Given a passage of natural language text, sentences that may possibly contain causal relations should be extracted. The text passage is parsed into sentences using the Stanford Parser ${ }^{2}$. A sentence parser is needed, since the mere presence of a period does not mark the end of a sentence, e.g., a period in an abbreviation.

If a sentence contains a verb from our dictionary of causal verbs (Table 3.1), then the sentence has a possibility of containing a causal relation that we can extract. However, just because a sentence includes one of our causal verbs does not necessarily mean it is causal. Therefore a method of filtering causal and non-causal sentences is needed.

\footnotetext{
${ }^{2}$ http://nlp.stanford.edu/software/lex-parser.shtml
} 
Verbs

\begin{tabular}{|c|c|c|c|c|}
\hline $\begin{array}{l}\text { actuate } \\
\text { actuates } \\
\text { actuating } \\
\text { affect } \\
\text { affecting } \\
\text { affects } \\
\text { breed } \\
\text { breeding } \\
\text { breeds } \\
\text { bring } \\
\text { bringing } \\
\text { brings } \\
\text { call forth } \\
\text { calling forth } \\
\text { calls forth } \\
\text { cause } \\
\text { causes } \\
\text { causing } \\
\text { compel } \\
\text { compelling } \\
\text { compels } \\
\text { complicate } \\
\text { complicates } \\
\text { complicating } \\
\text { decide } \\
\text { decides } \\
\text { deciding } \\
\text { decrease } \\
\text { decreases } \\
\text { decreasing } \\
\text { determine } \\
\text { determines } \\
\text { determining } \\
\text { dictate } \\
\text { dictates } \\
\text { dictating }\end{array}$ & $\begin{array}{c}\text { do } \\
\text { does } \\
\text { doing } \\
\text { effect } \\
\text { effecting } \\
\text { effects } \\
\text { effectuate } \\
\text { effectuates } \\
\text { effectuating } \\
\text { encourage } \\
\text { encourages } \\
\text { encouraging } \\
\text { engender } \\
\text { engendering } \\
\text { engenders } \\
\text { evoke } \\
\text { evokes } \\
\text { evoking } \\
\text { facilitate } \\
\text { facilitates } \\
\text { facilitating } \\
\text { force } \\
\text { forces } \\
\text { forcing } \\
\text { get } \\
\text { gets } \\
\text { getting } \\
\text { has } \\
\text { have } \\
\text { having } \\
\text { impel } \\
\text { impelling } \\
\text { impels } \\
\text { impose } \\
\text { imposes } \\
\text { imposing }\end{array}$ & $\begin{array}{c}\text { incite } \\
\text { incites } \\
\text { inciting } \\
\text { increase } \\
\text { increases } \\
\text { increasing } \\
\text { induce } \\
\text { induces } \\
\text { inducing } \\
\text { influence } \\
\text { influences } \\
\text { influencing } \\
\text { initiate } \\
\text { initiates } \\
\text { initiating } \\
\text { inspire } \\
\text { inspires } \\
\text { inspiring } \\
\text { instigate } \\
\text { instigates } \\
\text { instigating } \\
\text { kick up } \\
\text { kicking up } \\
\text { kicks up } \\
\text { kill } \\
\text { killing } \\
\text { kills } \\
\text { lead } \\
\text { leading } \\
\text { leads } \\
\text { let } \\
\text { lets } \\
\text { letting } \\
\text { make } \\
\text { makes } \\
\text { making }\end{array}$ & $\begin{array}{c}\text { mold } \\
\text { molding } \\
\text { molds } \\
\text { motivate } \\
\text { motivates } \\
\text { motivating } \\
\text { move } \\
\text { moves } \\
\text { moving } \\
\text { obligate } \\
\text { obligates } \\
\text { obligating } \\
\text { oblige } \\
\text { obliges } \\
\text { obliging } \\
\text { occasion } \\
\text { occasioning } \\
\text { occasions } \\
\text { persuade } \\
\text { persuades } \\
\text { persuading } \\
\text { pioneer } \\
\text { pioneering } \\
\text { pioneers } \\
\text { play } \\
\text { playing } \\
\text { plays } \\
\text { promote } \\
\text { promotes } \\
\text { promoting } \\
\text { prompt } \\
\text { prompting } \\
\text { prompts } \\
\text { propel } \\
\text { propelling } \\
\text { propels }\end{array}$ & $\begin{array}{l}\text { provide } \\
\text { provides } \\
\text { providing } \\
\text { provoke } \\
\text { provokes } \\
\text { provoking } \\
\text { reduce } \\
\text { reduces } \\
\text { reducing } \\
\text { regulate } \\
\text { regulates } \\
\text { regulating } \\
\text { result } \\
\text { resulting } \\
\text { results } \\
\text { set up } \\
\text { sets up } \\
\text { setting up } \\
\text { shape } \\
\text { shapes } \\
\text { shaping } \\
\text { solicit } \\
\text { soliciting } \\
\text { solicits } \\
\text { spawn } \\
\text { spawning } \\
\text { spawns } \\
\text { stimulate } \\
\text { stimulates } \\
\text { stimulating } \\
\text { suborn } \\
\text { suborning } \\
\text { suborns }\end{array}$ \\
\hline
\end{tabular}

Table 3.1: List of Verbs 


\subsubsection{Sentence Filtering}

Since extracting causes and effects from non-causal sentences would result in extraction errors, a method for filtering out non-causal sentences is needed. The method presented here uses a Fisher classifier that was trained with annotated training data to filter out non-causal sentences to help boost the precision of the system.

Given an extracted sentence, a Fisher classifier determines whether the sentence is causal or not. 1,658 sentences were hand-annotated for the purpose of training a Fisher classifier to decide if a given sentence is causal or not. Through empirical testing, filtering out non-causal sentences not only helped to reduce the amount of computation performed during the tuple extraction step, but also worked to increase the precision of the system (with a possible harm to the recall). The reason for this is intuitive, since if the system were to extract causes and effects from a sentence that is not causal, then that extraction would be false.

The Fisher classifier used was part of the ci-bayes ${ }^{3}$ library and is based on the Fisher classification algorithm from [40]. Fisher classifiers use learned probabilities about features which occur in a training set to classify an input into one of the trained categories [40]. For the causal Fisher classifier, the following features were considered:

1. Filtering out common "stop" words (c.f. Table 3.2).

2. Mark increasing words with inc marker and include the word.

3. Mark decreasing words with _dec marker and include the word.

4. Mark causal verbs with _verb tag and include the verb.

\footnotetext{
${ }^{3}$ https://ci-bayes.dev.java.net/
} 
Stop Words

\begin{tabular}{|c|c|c|c|c|}
\hline a & etc & like & that & wants \\
about & example & may & thats & we \\
also & for & me & the & were \\
among & from & more & their & when \\
an & has & much & them & where \\
and & he & my & themselves & which \\
another & her & of & then & while \\
any & herself & on & there & who \\
are & himself & or & these & whom \\
as & his & other & they & will \\
at & how & others & thing & with \\
be & i & our & things & would \\
because & if & she & this & you \\
been & in & so & those & your \\
being & into & some & through & \\
both & is & someone & to & \\
but & it & something & today & \\
by & its & such & var & \\
can & itself & than & very & \\
\hline
\end{tabular}

Table 3.2: List of Stop Words

5. Add in stems of the words in the sentence.

6. Verb patterns plus the phrase "verbPatt."

7. Word patterns plus the phrase "wordPatt."

The increasing and decreasing words considered as features were simply taken as a closed, arbitrary set of words with "increasing" and "decreasing" connotations. The increasing words used for our set are shown in Table 3.3 and the decreasing words are shown in Table 3.4. For future work, research should be done as to what other words should be included in these sets.

Through empirical testing of all $\left(2^{7}=\right) 128$ possible combinations, it was found that training the classifier with the following features worked best:

1. Filtering out common "stop" words (c.f. Table 3.2). 
Increasing Words

\begin{tabular}{|c|c|c|}
\hline benefit & high & rise \\
benefits & higher & rises \\
contribute & improve & rising \\
contributed & improves & significant \\
contributes & increase & strength \\
fuelling & increases & strengthen \\
further & increasing & strengthening \\
furthering & leads & strengthens \\
furthers & more & strengths \\
grow & positive & surge \\
growth & positives & surges \\
\hline
\end{tabular}

Table 3.3: List of Increasing Words

Decreasing Words

\begin{tabular}{|c|c|c|c|}
\hline cut back & higher decreases & move away & reductions \\
cut backs & immunize & moves away & remove \\
decrease & immunizes & move from & removes \\
decreases & immunizing & moves from & suppress \\
decreasing & is not & negative & suppresses \\
decreasings & knocks off & negatively & unlikelies \\
fall & knock offs & negatives & unlikely \\
fallen & less & no & weak \\
falling & lesser & not all & weaken \\
falls & lessens & not so & weakening \\
few & little & reduce & \\
fewer & littles & reduces & \\
get out & low & reducing & \\
gets out & lower & reducings & \\
higher decrease & lowers & reduction & \\
\hline
\end{tabular}

Table 3.4: List of Decreasing Words 
2. Mark decreasing words with_dec marker and include the word.

3. Mark causal verbs with _verb tag and include the word.

4. Verb patterns plus the phrase "verbPatt."

5. Word patterns plus the phrase "wordPatt."

This filter on the sentences not only helped to reduce the amount of computation performed later on in the tuple extraction, but also worked to increase the precision of the system (with a slight harm to the recall), as shown in our results (Chapter 5).

\section{Verb and Word Pattern Generation}

The verb patterns and word patterns used by the Fisher classifier in the sentence filtering step (Section 3.4.3) were generated in the following way. First, 1,658 sentences were annotated, out of which 500 were causal. For each annotated sentence that was causal, n-grams of words both before and after the causal verb in the sentence (if it existed), with a maximum of five words $(n=5)$ on each side

of the verb, were saved to the database. The frequency of each pattern was also recorded, in order to determine which patterns were more important than others, though this information was not used here.

For the word patterns the same methodology was used, but instead of storing n-grams around causal verbs, we stored n-grams around domain terms that appeared in the annotated sentences. 


\subsubsection{Tuple Extraction}

To extract tuples in the form shown in Figure 3.1, a grammar-based extraction method is used.

The grammar-based causal relation extraction method uses the Stanford Parser ${ }^{4}$ in order to build a parse tree of a sentence. The parse tree is then used to identify the causes and effects in the sentences in a way similar to that of Khoo et al. [18], who also used parse information to identify causes and effects. Since our list of verbs (see Table 3.1) is a closed-set that typically takes causes on the lefthand side of the verb and effects on the right-hand side, we use that information to extract causes and effects from the parse tree. Since human natural language text can often be quite complex in its structural patterns, we limit the grammar-based extraction pattern to only a very simple pattern where a noun-phrase is followed by a verb-phrase, that is contained in a noun-phrase that is dependent on the verb phrase. An example of this pattern is shown in Figure 3.3.

Specifically, our extraction pattern looks for a tag in the parse tree that begins with "VB," which denotes that the word or words within the tag are Figure 3.3: Parse Tree

\footnotetext{
${ }^{4}$ http://nlp.stanford.edu/software/lex-parser.shtml
}

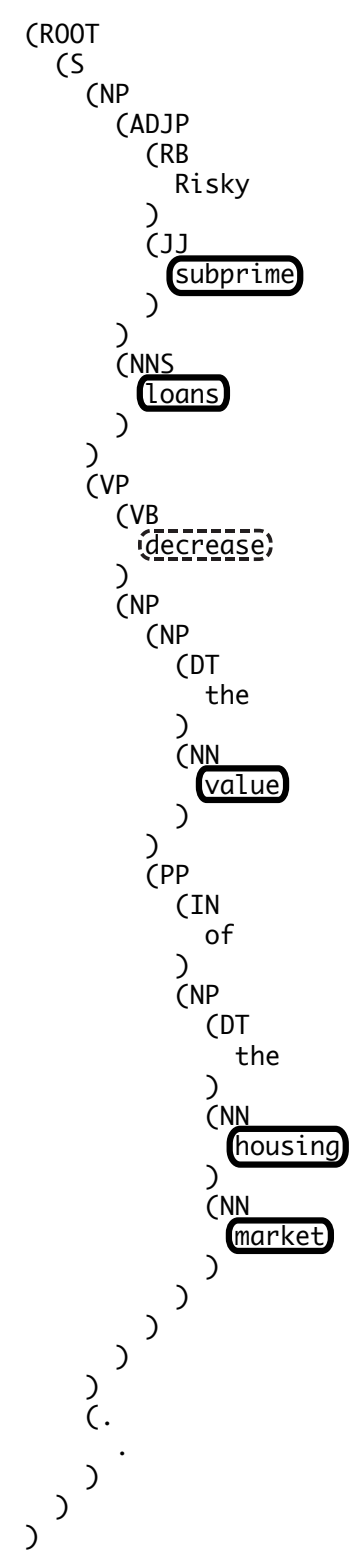


verbal. If the word in the VB tag is in our list of causal verbs, then we go before the VB tab until we find a tag that begins with "NP," denoting a noun phrase. A cause is then extracted from nested tags beginning with JJ (adjectives) and NN (nouns).

The extraction of causes also considers conjunctions and disjunctions, c.f., Sanchez-Graillet and Poesio [39]. Conjunctions are handled such that if a conjunction is found nested in a noun phrase (NP tag), then the tags beginning with JJ and NN are added together with a comma separating the different parts, so that multiple nouns become one cause that causes an effect. For example, given the conjunctive sentence, "Subprime lending and deregulation decrease the value of the housing market," the following tuple would be extracted: $<$ [subprime lending, deregulation], decrease, value housing market, negative $>$.

Disjunctions are handled such that if a disjunction is found nested in a noun phrase (NP tag), then the tags beginning with JJ and NN are extracted as separate causes, each having the same effect. For example, given the disjunctive sentence "Subprime lending or deregulation decreases the value of the housing market," the following tuples would be extracted: <subprime lending, decreases, value housing market, negative $>$ and $<$ deregulation, decreases, value housing market, negative $>$.

Putting it all together, given the sentence, "Risky subprime loans decrease the value of the housing market," the parse tree shown in Figure 3.3 would be generated by the grammar parser. Then the causal verb that is circled with a dashed line in the figure would be found. The circled words before the causal verb in the figure would be extracted as the cause, whereas the circled words after the causal verb are the effect. After the extraction of the cause and effect are complete, the polarity of the extracted relation must be classified. 


\subsubsection{Polarity Classification}

Out of 1,658 sentences that were annotated for training the Fisher classifier, 500 were causal and thus had polarity annotations. As with the Fisher classifier for filtering out non-causal sentences (Section 3.4.3), the ci-bayes library was used to perform the polarity classification. Though conceptually polarity can be considered to be positive, negative, or neutral, it was found that out of the 500 annotated sentences with polarity information, only twelve were annotated with neutral polarity, which was not enough to obtain good results from the Fisher classifier. Therefore, only two categories of polarity classification were considered: positive and negative. This is reasonable since causal relations are rarely considered to be neutral, since causal relations, by their nature, affect a system.

In training the Fisher classifier for polarity, we considered the same seven features that were considered in training the causal classifier:

1. Filtering out common "stop" words.

2. Mark increasing words with inc marker and include the word.

3. Mark decreasing words with _dec marker and include the word.

4. Mark causal verbs with _verb tag and include the verb.

5. Add in stems of the words in the sentence.

6. Verb patterns plus the phrase "verbPatt."

7. Word patterns plus the phrase "wordPatt."

Through empirical testing of all $\left(2^{7}=\right) 128$ possible combinations, it was found that training the classifier with the following features worked best: 
1. Mark increasing words with inc marker and include the word.

2. Mark decreasing words with_dec marker and include the word.

3. Mark causal verbs with _verb tag and include the verb.

4. Add in stems of the words in the sentence.

\subsubsection{Tuple Scoring}

After a set of tuples has been extracted, the phrases in causes and effects can be scored. A list of the phrases from all extracted causes and effects is then built. An example of a phrase would be "subprime lending" if that was either a cause or an effect in a tuple. Each phrase is scored by determining the frequency, raising the frequency of the phrase to the power of three, and adding to that number the number of words in the phrase. This puts a high emphasis on the frequency of phrases, but also allows the length of the phrases to have some effect. When building the causal-association network, combinations of causes and effects that have a term with a score below a predetermined threshold are automatically ignored. This is in keeping with our governing assumption (c.f. Section 3.1) that if many people describe a situation a certain way, then perhaps it is an accurate, or at least useful, view of the situation. Typically the threshold level is set such that more than $90 \%$ of the extracted tuples are used to build the network.

\subsubsection{Influence Calculation}

We generate a causal-association network from all the tuples of causes and effects that have both a cause and an effect that are above a certain score threshold, as determined in the tuple scoring step (Section 3.4.6). Since multiple tuples 
can have the same causes and effects or the causes and effects can be the opposite in some tuples than they are in other tuples, we have to calculate the net relation strength between pairs of causes and effects. We do this by taking a cause and effect and subtracting tuples which have the cause as the effect and the effect as a cause. We also add in tuples that have the same cause and effect. In this way a net connection strength between a cause and an effect is determined. We do this same calculation to determine the net polarity between a cause and an effect.

\subsection{Visualization}

Though causal-association networks can potentially be visualized any number of ways, in this section one way of displaying causal-association networks for easy consumption by humans is outlined.

Since causal-association networks lend themselves naturally to graphical visualization, a program was created that allows a causal-association network to be viewed and manipulated in order to facilitate understanding of a domain. Our visualization features a graph of phrases that are connected together in order to show their causal relations. If there is a net strength between one phrase on another, as determined in Section 3.4.7, then an arrow is drawn in the direction of the relation. The thickness of the connecting lines is a function of the net strength between the relations. In other words, the more data which states that two terms are causally connected in the net direction shown in the graph, the thicker the line will be. If the net polarity between two terms is positive, then the line will be red. If the net polarity between two terms is negative, then the line will be black. Neutral polarity, which can happen if the positive and negative polarities cancel each other out, is shown by a blue line. Drawing the visualization of the 


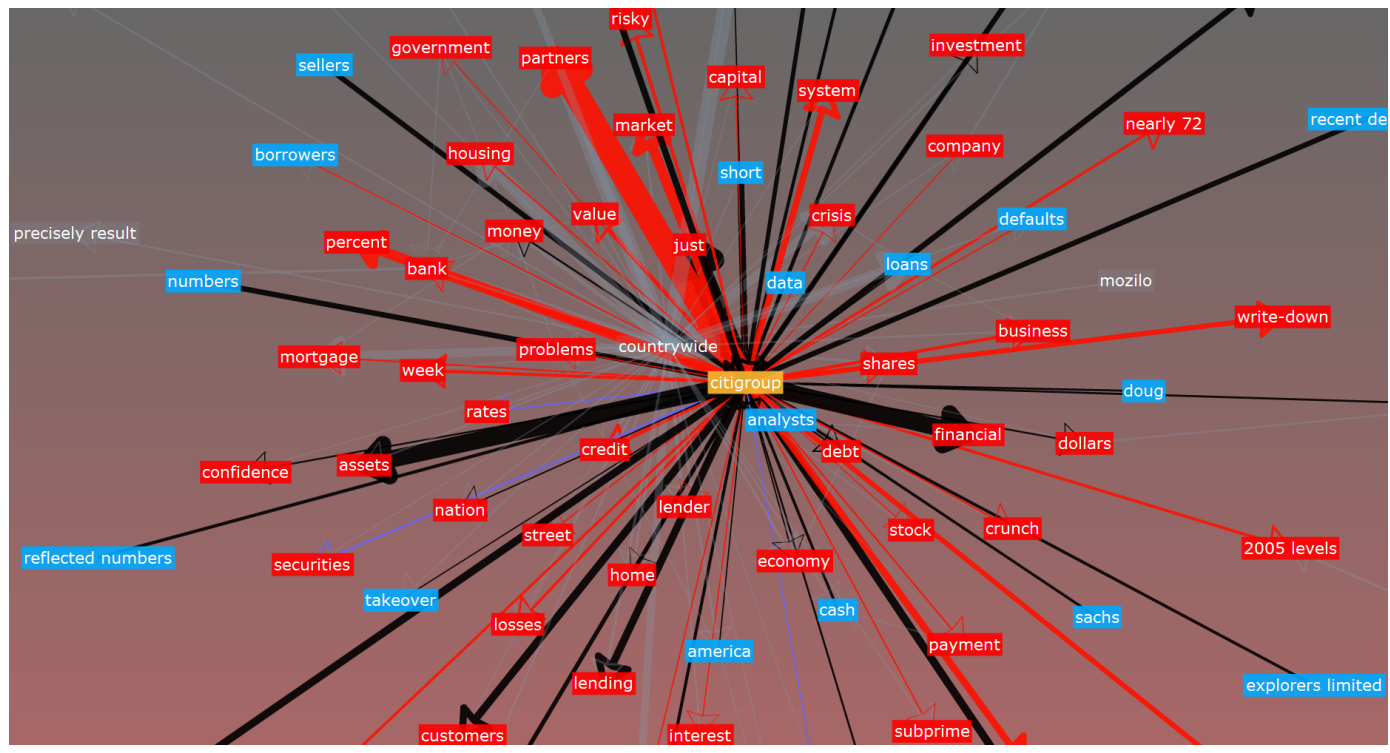

Figure 3.4: Visualization for Financial Domain

data in this way easily summarizes the information of the CAN so that a human will be able to look at the visualization as an aid to understanding the way in which variables in the domain they are working in are causally connected.

If the user of the visualization clicks on a node on the graph, then that node will be highlighted as yellow and the effects of that node will be highlighted as red, whereas the causes of the clicked node are highlighted as blue. To simplify the visualization, all the other nodes and lines will be made partially-transparent and grayed out. This is shown in Figure 3.4.

For future work, more features could be added to the visualization, such as sorting the nodes when you select a node so all the causes are on the left-hand side and all the effects on the right-hand side. Additionally, usability studies should be performed in order to determine how the visualization can be improved. 


\subsection{Summary Overview of Causal Association Networks}

Causal-association networks are a way to structure causal relations that are extracted from unstructured text data. They are generated from tuples of causes, causal verbs, effects, and polarity information. Due to the importance of causality (c.f. Section 1.1), it is useful to have causal relations in a form that can easily be used for either assisting human reasoning or automating the reasoning of intelligent software agents.

In this chapter a method for extracting causal-association networks (and thus tuples of causal relations) has been explicated. The presented methodology, the efficacy of which is measured in Chapter 5, uses a grammar parser to extract causes and effects from English natural language unstructured text data. This extraction is accomplished by using a simple grammar pattern, described in Section 3.4.4. In order to prevent causes and effects from being extracted from noncausal sentences, a classifier (c.f. Section 3.4.3) is used that filters out non-causal sentences so they are not processed.

Since polarity of causal relations is important to understanding how a cause determines an effect, a classifier, described in Section 3.3, was trained that will classify an extracted tuple as having either positive or negative polarity. Expanding causal-association networks to hold more data about causal relations is a project for future work. 


\section{Chapter 4}

\section{Framework for Extracting}

\section{Causal-Association Networks}

\section{from the Internet}

Causal-association networks are a way to represent a summary of the causal interactions between variables in a system. The purpose is to store causal relations that have been extracted from unstructured text data. As of this writing, a profoundly large amount of unstructured text data exists across web sites and networks on the Internet. If even a small fraction of this data is textual then it is still impossible for one person or even a good-sized team of people to read and summarize all the data. Therefore, automated methods are needed. To address this need, this chapter focuses on a methodology for extracting causal-association networks from the Internet.

Also, since much of the textual data on the Internet is in the form of personal opinions, extracting causal relations from this data may provide a method for 
analyzing the underlying sentiments of people writing on the internet and determining majority opinion. Applying causal-association networks to sentiment extraction is outside the scope of this thesis, but it is a possible topic for future work.

\subsection{Causal Tuple Extraction from the Internet}

In order to create a causal-association network, tuples from unstructured text data must first be extracted. These tuples are in the form shown in Figure 3.1, explained in Chapter 3. One tuple consists of a cause, a causal verb, an effect, and polarity information. Our emphasis on the representation of data as tuples instead of individual terms, as was done by Saito et al. [37] and Perrin et al. [30], is a notable distinction since this potentially allows for more meta-data (i.e., context) to be included with the the cause, causal verb, and effect. We do this by including direction and polarity information for the terms in the causal relation.

The causal tuple extraction process is a cyclical bootstrapping process that consists of several steps: query generation; sentence extraction; sentence filtering; causal tuple extraction; tuple scoring; and then the generation of more queries. An overview of the process of causal tuple extraction from the Internet is shown in Figure 4.1. As shown in the figure, extracting tuples from Internet data is a cycle that will never end as long as there are new results to new queries that are generated. However, a causal-association network can be created from the extracted tuples at any time by calculating the influences of the tuples, as discussed in Section 3.4.7.

As you will notice if you compare Figure 4.1 to the causal-association network generation (Figure 3.2), the process for extracting causal association networks is 


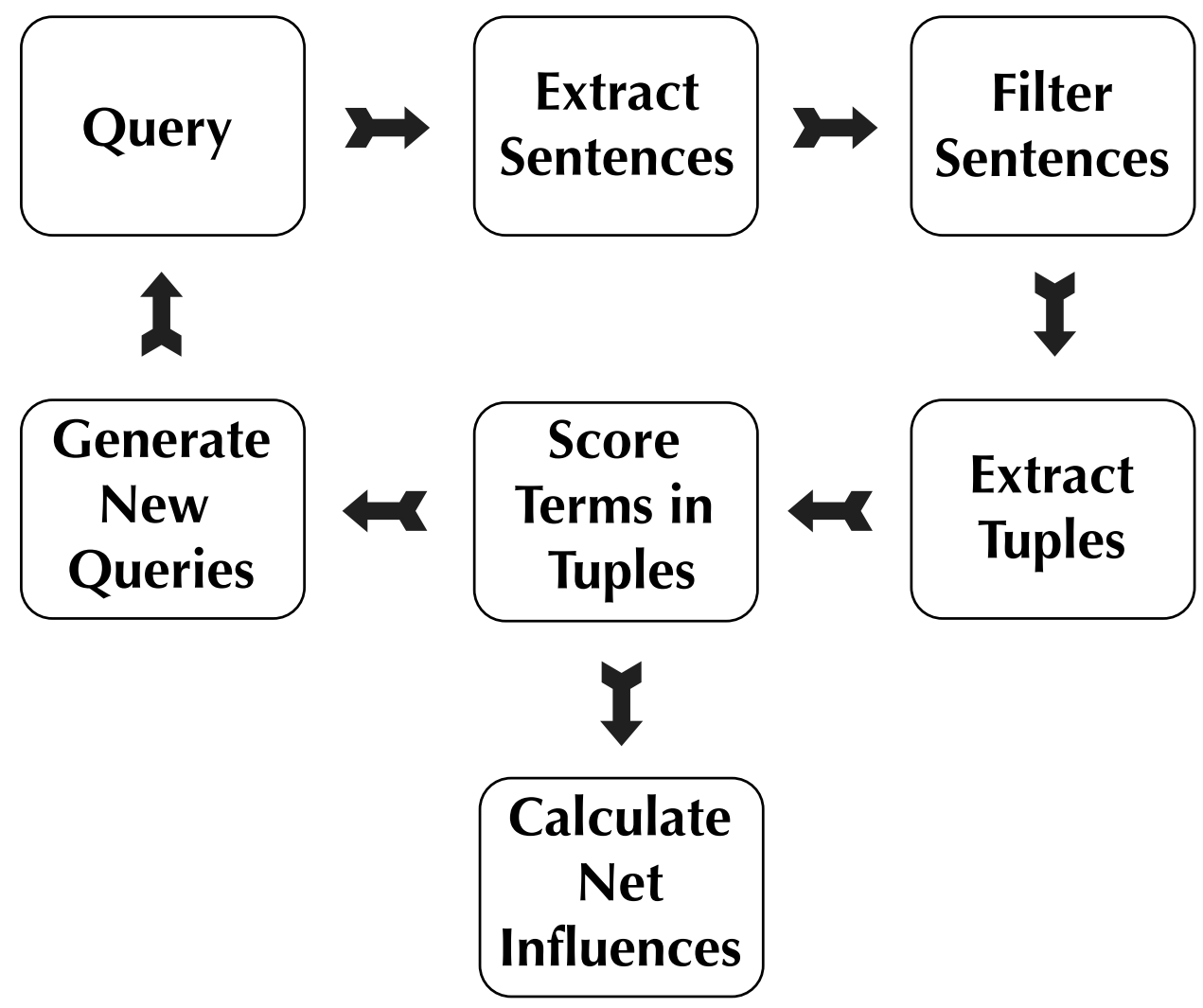

Figure 4.1: Overview of Causal Tuple Extraction 
the same on the Internet as from regular text data, only the Internet extraction process includes query and query generation steps. Since the other steps are the same, we only need discuss the query and query generation steps here. For a complete description of the other steps, please see Chapter 3. The following sections will describe this process in more detail.

\subsubsection{Querying for Text Data}

Queries are executed on the Yahoo! search $\mathrm{API}^{1}$ and a maximum of 100 URLs for each query are saved. To generate the queries, two dictionaries are utilized: a dictionary of causal verbs and a dictionary of domain terms. A list of all the verbs we use appears in Table 3.1. Domain terms are terms like "subprime loans" that are significant terms in some domain. To start the extraction process, a list of these terms must be provided by the user. For all terms and causal verbs, the following query patterns can be generated:

- "TERM * VERB"

- "VERB * TERM"

Where the asterisk denotes a wild-card character, meaning any number of any characters, and the quotation marks around the query show that it is sent as a quoted phrase to the Yahoo! search API. This is important because we want to allow some variation in terminology between the term and the causal verb while also guaranteeing that the verb and the term we are searching for actually appear in their complete forms, since the term could be a multi-word phrase. For

\footnotetext{
${ }^{1}$ http://developer.yahoo.com/
} 
example, if you have the term, "oil prices," and the causal verb, "cause," then the generated queries would be:

- "oil prices * cause"

- "cause * oil prices"

When these queriers are executed, then the Yahoo! search API will return up to 100 URLs for each query that references web pages that contain both the phrase "oil prices" and the word "cause." Once the URLs are retrieved from the search API, the text on the referenced web pages needs to be analyzed and sentences containing both the term and the verb we are searching for need to be extracted.

\subsubsection{Sentence Extraction from the Internet}

For each URL retrieved by querying the Yahoo! search $\mathrm{API}^{2}$, the text is parsed into sentences using the Stanford Parser ${ }^{3}$. As with extracting tuples from non-Internet data, a sentence parser is needed since the mere presence of a period does not mark the end of a sentence, e.g., a period in an abbreviation. Extracting causal tuples from the Internet is somewhat more complex than for text data that comes from other sources that do not have embedded formatting, since HTML and JavaScript formatting need to be stripped away. Fortunately, the Stanford Parser also strips out all the HTML formatting on HTML pages. After this is done, each sentence that contains both a term from the dictionary of domain terms and a verb from the dictionary of causal verbs (c.f. Table 3.1) is saved to a database containing possibly causal sentences. These sentences can then

${ }^{2}$ http://developer.yahoo.com/

${ }^{3}$ http://nlp.stanford.edu/software/lex-parser.shtml 
be filtered so that non-causal sentences can be taken out, and the process of generating a causal-association network continues as normal until we are ready to generate new queries.

\subsection{New Query Generation}

The process of causal tuple extraction from the Internet is cyclical and follows a bootstrapping cycle since the highest-scoring domain terms that exist in the extracted tuples are then used to generate more queries. In this way, the terms that are first input to generate the queries are then branched-out in a process similar to bootstrapping, in which a small amount of seed terms expand into a larger list of terms, based on the co-occurrence in the data (c.f. [17]).

\subsection{Summary of Extracting Causal-Association Networks from the Internet}

Causal-association networks can be used to summarize a large amount of causal relations in a form that is readily accessible to a human. Since the Internet has a very large number of unstructured text data, this chapter has presented a methodology explicating how causal-association networks can be extracted from the Internet using a cyclical process to query using a set of terms and then find new terms to query with, thus increasing the amount of causal relations that are extracted. 


\section{Chapter 5}

\section{Verification and Results}

Thus far an extraction methodology for extracting tuples of causal relations from unstructured text data has been presented. In this process there are three steps that must be verified in order to determine the efficacy of the approaches: causal/non-causal sentence classification; polarity classification; and cause and effect extraction. In Section 5.1 metrics are presented for verifying the approaches used.

\subsection{Metrics}

The generation of causal-association networks is comprised of several steps that need to be evaluated, namely the binary causal classification (Section 3.4.3), the causal tuple extraction (Section 3.4.4), and positive and negative polarity classifications (Section 3.4.5). Since there is no standard data set that we can use to compare our approach to other approaches, we need to use some kind of quantitative metrics to evaluate our results. To this end we computed the precision, recall, and F-score for the classifiers and the causal tuple extraction. 
These metrics are commonly used in information extraction tasks and much of the related work (c.f. Chapter 2) use these metrics.

Precision is a measure of how precise, or accurate, the results are. We define precision to be: (\# correct classifications) / (\# correct classifications + \# incorrect classifications). Recall is a measure of how many correct extractions out of the total number of possible correct extractions are extracted. Recall is defined to be: (\# correct classifications) / (\# classifications for the category).

Precision and recall are often inversely related to each other, since one can get a perfect precision by simply recalling nothing or, conversely, one can get a perfect recall by simply extracting everything. In order to balance these competing metrics, an F-score is typically computed. The F-score is defined as the harmonic mean between precision and recall, i.e, $(2 *$ precision $*$ recall $) /$ $($ precision + recall $)$. Thus the F-score is a good measure to see how well an extraction method works overall.

\subsection{Causal and Polarity Classification Results}

For the causal classification a fifteen-fold cross-validation on the annotated training set was performed, using 1,500 of the annotated sentences. The result of the cross-validation tests were a precision of $71.3 \%$, recall of $94.6 \%$, and an F-score of $81.3 \%$.

For the positive and negative polarity classifications, a five-fold cross-validation was performed on the annotated training set of 500 causal sentences. Using the Fisher classifier, a precision of $72.1 \%$, recall of $80.2 \%$, and an F-score of $75.97 \%$ 
were obtained. Again, since a system can get higher precision by lowering the recall or get a lower precision but raise the recall, the F-score is a good way of balancing the two metrics.

\subsubsection{Sources of Error}

Fisher classifiers typically do best with symmetrical training sets where equal amounts of annotated training data exists for each category that is being classified. However, the training sets for the classifiers were not symmetrical. The causal classifier had 1,000 negative examples of non-causal sentences, but only 500 causal sentences.

For the polarity classifier, only 145 sentences out of 500 had negative polarity. Thus for future work, if symmetric training sets for the Fisher classifiers were created, then better results are likely to be obtainable.

\subsection{Causal Tuple Extraction Results}

From the PubMed web site ${ }^{1}$, all the abstracts of papers relating to "pandemic influenza" were extracted on May 5, 2009, and saved to a database. In total, 3,408 abstracts were saved. Out of the 3,408 abstracts, one hundred abstracts were randomly chosen and hand-annotated for cause and effect information. The results of running our extraction method on the annotated data are shown in Table 5.1.

As shown in the extraction results, adding the causal filter helps to increase the precision by $6.28 \%$, while only lowering the recall by $0.58 \%$. Overall, this

\footnotetext{
${ }^{1}$ http://www.ncbi.nlm.nih.gov/pubmed/
} 
Causal Tuple Extraction

\begin{tabular}{|c|c|c|c|c|c|}
\hline \multicolumn{3}{|c|}{ No Causal Filtering } & \multicolumn{3}{c|}{ Causal Filtering } \\
\hline Precision & Recall & F-Score & Precision & Recall & F-Score \\
\hline $79.63 \%$ & $74.57 \%$ & $77.02 \%$ & $85.91 \%$ & $73.99 \%$ & $79.5 \%$ \\
\hline
\end{tabular}

Table 5.1: Causal Tuple Extraction Results

increases the F-score of the approach that filters out non-causal sentences by $2.48 \%$. This would suggest that there is indeed merit for filtering out non-causal sentences before attempting a causal extraction process. A graph of some of the causal relations extracted via the filtered method is shown in Figure 5.1.

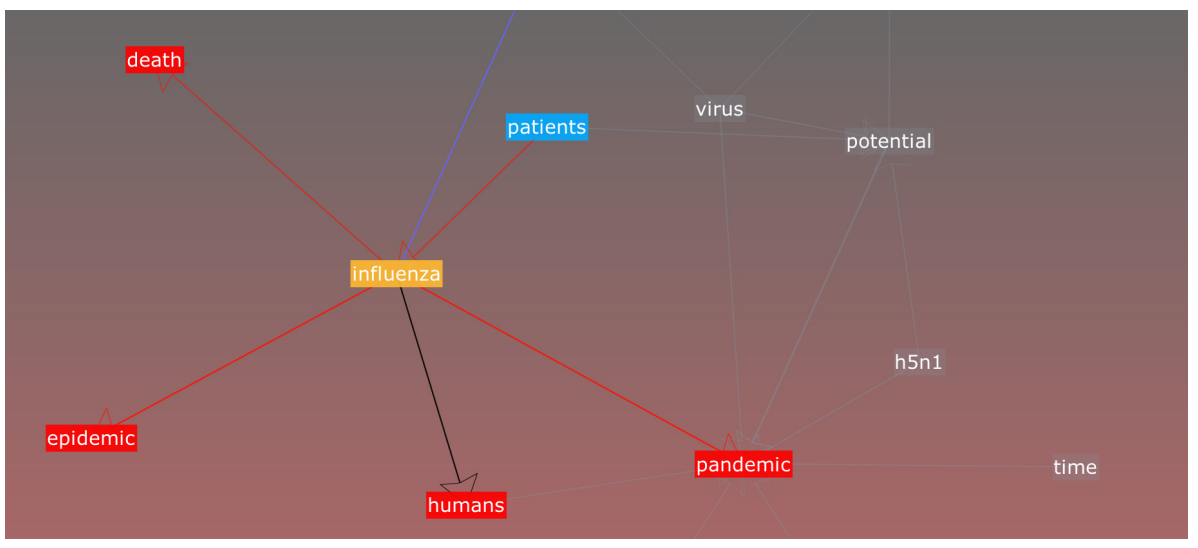

Figure 5.1: Visualization for Extracted Medical Abstract Data

\subsubsection{Sources of Error}

There are several sources of error for the causal tuple extraction process, the main ones being: an insufficiently expressive grammar extraction pattern; anaphora resolution; incorrect level of specificity; and ignoring negation, thereby extracting negated causal relations.

One of the main factors affecting the recall of the causal relation extraction is that in order to extract a tuple from a sentence, the cause and effect must be represented in the sentence such that the grammar pattern used can extract it. 
Since the grammar pattern used in this thesis is fairly simple, future work can most assuredly improve on the recall of the extraction by expanding the grammar pattern used.

Anaphora resolution, such as resolving pronoun anaphora, is an open problem in natural language processing [4], where one word, such as "he" or "it" references another word. In many cases, calculating the word that is being referenced is hard to do precisely, due to ambiguity in language. The system presented in this thesis ignores this problem altogether, so valid causal relations are likely to be missed, thus hurting recall, since the extraction pattern only extracts nouns (NN tags from the parser) and adjectives (JJ tags).

Another source of error in the causal extraction process is extracting nouns or adjectives and nouns that are at the incorrect level of specificity. For example, possible ways of saying the same type of "bird flu" would be: "influenza," "avian influenza," "highly pathogenic avian influenza," "H5N1 avian influenza," or "HPAI H5N1 avian influenza." Depending on the use(s) for the extracted causal relations, different levels of specificity should desired.

Finally, yet another source of error in our system is extracting causal relations that have been negated within a sentence. A good example of this is with the following sentence from the data set used to test the causal relation extraction:

However, aluminum-adjuvant but not MF59-adjuvant combined vaccination was able to induce high titers of anti-M2e antibodies and provoke M2e-specific T lymphocyte response. (sentence extracted from the abstract of [50])

The extracted causal tuple for this sentence is: <aluminum-adjuvant mf59adjuvant combined vaccination, induce, high titers anti-m2e antibodies, positive $>$, using our standard tuple format explicated earlier. This is obviously wrong, since 
only the aluminum-adjuvant vaccination should have been extracted as a cause. Thus ignoring negated causal relations can have a detrimental effect upon the precision of the system.

\subsection{Summary of Results}

In order to analyze the efficacy of the methodology presented in this thesis, three parts of the system needed to be verified: the causal/non-causal sentence classification, the polarity classification, and the causal relation extraction (extraction of causes and effects). Precision, recall, and F-score statistics were computed for each verified part of the system, in order to allow some comparison with related works, though there was no standard data set in the causal relation extraction domain that we could use.

Of particular interest are the results of the causal relation extraction. This part of the system was verified by annotating abstracts relating to "pandemic influenza" that were taken from the PubMed ${ }^{2}$ website. The computed precision, recall, and F-scores for both the causal relation extraction method without causal/non-causal sentence filtering and the method with the filtering are fairly close, but there is a higher precision and higher F-score for the causal extraction method with the filter to remove non-causal sentences. This is verification that there is merit in filtering out non-causal sentences before beginning a causal relation extraction process. Thus future work should focus on expanding and improving the methodology used for accomplishing this filtering of sentences.

\footnotetext{
${ }^{2}$ http://www.ncbi.nlm.nih.gov/pubmed/
} 


\section{Chapter 6}

\section{Conclusions and Future Work}

Causality is important to understanding the dynamics between variables in a domain. In this thesis a system was presented that extracts explicitly-defined causal relations from unstructured English natural-language text. The system uses the extracted relations to create a causal-association network that can be used to facilitate decision-making by domain experts. While the experiments so far have been somewhat limited (mostly due to the need to manually annotate sentences with respect to causality), the results indicate that the approach used is conceptually sound. We believe that some improvements can be made through modifications of the algorithms and methods used here, but in order to make significant progress, future work is needed to extract causes and effects which are only implicitly expressed in unstructured text data. The recognition and interpretation of such implicit causality is easy for humans, but remains a major challenge for computers. 


\subsection{Conclusion}

This thesis presents a system for the extraction of causal-association networks from unstructured text on the Internet. A causal-association network is a network

of directed causal relations that have polarity determined as being either positive or negative. Networks of causes and effects such as this can be used to aid human decision-makers, since causal relations express the dynamics of variables within a domain influencing each other.

The polarity of a causal relation, a part of the extracted causal tuples that make up causal-association networks, is a measure of how a cause affects an effect. This thesis only considers positive and negative polarity, but future work should certainly try to expand this concept more to include other features.

Finally, in addition to considering the concept of polarity, another notable aspect of the system presented in this thesis is the filtering of sentences such that non-causal sentences can be removed. Doing this, as shown in the results (Chapter 5), can help increase the precision of the system by preventing the erroneous extraction of causes and effects from non-causal sentences.

\subsection{Future Work}

As no project is ever truly complete, there are naturally some directions for further research. One good next step would be to split up the causal-association network generation process (c.f. Figure 3.2) so that collaborative agents can work together to divide and conquer the tasks so the network can be added to and updated continuously in real-time.

Also, the precision and recall of the causal/non-causal classifier and the po- 
larity classifier should be improved upon. Future work may also consider other approaches, such as support vector machines [6] to perform this classification.

Furthermore, for the polarity classifier, sentence-level granularity for polarity classification is inadequate, since multiple causal relations can be in one sentence and have different polarities. Future work should focus on trying to classify the polarities of individual causal relations within a sentence.

Finally, in order to boost the recall of the cause and effect extractions, work should be done on expanding the grammar patterns used so that features such as negations of causal relations within a sentence can be taken into account.

\subsubsection{List of Open Problems}

Oftentimes the open problems in a field of study are not readily apparent. In order to promote further research and advancement in the study of causalassociation networks, the following enumerated list of open problems is presented. This list is provided with the sincere hope that these open problems can be worked on by many people and that their resolution will aid the understanding of our world and the fulfillment of human potential.

1. Expand the cause and effect extraction methodology so that a grammar parser is not needed and thus the extraction method is language-independent.

2. Use causal knowledge for the automatic creation of ontological data for agent-based reasoning.

3. Expand the grammar-based extraction method to include more patterns.

4. Use different representations for text to allow for implicit, as well as explicit, causation to be extracted. 
5. Create an auto-associative representation of text that will allow for autoassociative and self-organizing extraction of causal relations. 


\section{Bibliography}

[1] Bayes, T. An Essay Towards Solving a Problem in the Doctrine of Chances. Published posthumously in. Phil. Trans. Roy. Soc. London 53 (1763), 370418.

[2] Brooks, R. Intelligence Without Reason. In Proceedings of the 12th International Joint Conference on Artificial Intelligence (IJCAI-91) (1991), pp. 569-595.

[3] Charniak, E. Bayesian Networks without Tears. AI Magazine 12, 4 (1991), $50-63$.

[4] Charniak, E., And Elsner, M. EM Works for Pronoun Anaphora Resolution. In Proceedings of the Conference of the European Chapter of the Association for Computational Linguistics (EACL-09), Athens, Greece (2009), pp. $148-156$.

[5] Cooper, G. The Computational Complexity of Probabilistic Inference Using Bayesian Belief Networks. Artificial Intelligence 42 (1990), 393-405.

[6] Cristianini, N., and Shawe-Taylor, J. An Introduction to Support Vector Machines. Cambridge University Press, 2000. 
[7] Dawid, A. Influence Diagrams for Causal Modelling and Inference. International Statistical Review 70, 2 (2002), 161-189.

[8] Fellbaum, C. WordNet: An Electronic Lexical Database. MIT Press, 1998.

[9] Girju, R. Automatic Detection of Causal Relations for Question Answering. In Proceedings of the ACL 2003 Workshop on Multilingual Summarization and Question Answering-Volume 12 (2003), Association for Computational Linguistics, Morristown, NJ, USA, pp. 76-83.

[10] Giruu, R., and Moldovan, D. Text Mining for Causal Relations. In Proceedings of the FLAIRS Conference (2002), pp. 360-364.

[11] Higashinaka, R., And Isozaki, H. Automatically acquiring causal expression patterns from relation-annotated corpora to improve question answering for why-questions. ACM Transactions on Asian Language Information Processing (TALIP) 7, 2 (2008), 1-29.

[12] Hoover, K. Causality in Macroeconomics. Cambridge University Press, 2001.

[13] Hoover, K. Causality in economics and econometrics. In The New Palgrave Dictionary of Economics Online, S. Durlauf, L. E. Blume, and P. Macmillan, Eds. Palgrave Macmillan, 2009.

[14] IWASAKI, Y. On the relationship between model abstraction and causality: variance of causal ordering under abstraction operations. In Pacific Rim International Conference on Artificial Intelligence (1990).

[15] Imasaki, Y., And Simon, H. Causality in device behavior. Artificial Intelligence 29, 1 (1986), 3-32. 
[16] Iwasaki, Y., And Simon, H. Causality and Model Abstraction. Artificial Intelligence 67, 1 (1994), 143-194.

[17] Kawai, H., Mizuguchi, H., And Tsuchida, M. Cost-Effective Web Search in Bootstrapping for Named Entity Recognition. Lecture Notes in Computer Science 4947 (2008), 393.

[18] Khoo, C. S. G., Chan, S., And Niu, Y. Extracting causal knowledge from a medical database using graphical patterns. In In Proceedings of 38th Annual Meeting of the ACL, Hong Kong (2000), pp. 336-343.

[19] Kudo, T., And Matsumoto, Y. A boosting algorithm for classification of semi-structured text. In Conference on Empirical Methods in Natural Language Processing (EMNLP) (2004).

[20] Kulikowski, C. A., And Weiss, S. M. Representation of expert knowledge for consultation: The CASNET and EXPERT projects. In Artificial Intelligence in Medicine, P. Szolovits, Ed. Westview Press, Boulder, Colorado, 1982, ch. 2 .

[21] Lang, J., Lin, F., And Marquis, P. Causal Theories of Action: A Computational Core. In Proceedings of the Eighteenth International Joint Conference on Artificial Intelligence (IJCAI-03) (2003), vol. 14, pp. 10731078.

[22] Lin, F. Embracing Causality in Specifying the Indirect Effects of Actions. In Proceedings of the Fourteenth International Joint Conference on Artificial Intelligence (IJCAI-95) (1995), vol. 2, pp. 1985-1993. 
[23] McCain, N., And Turner, H. A Causal Theory of Ramifications and Qualifications. In Proceedings of the Fourteenth International Joint Conference on Artificial Intelligence (IJCAI-95) (1995), vol. 14, pp. 1978-1984.

[24] McCain, N., And Turner, H. Causal Theories of Action and Change. In Proc. AAAI (1997), vol. 97, pp. 460-465.

[25] McCarthy, J., and Hayes, P. J. Some philosophical problems from the standpoint of artificial intelligence. In Machine Intelligence 4, B. Meltzer and D. Michie, Eds. Edinburgh University Press, 1969, pp. 463-502.

[26] Oldershaw, R. Democritus-Scientific Wizard of the 5th Century BC. Speculations in Science and Technology 21, 1 (1998), 37-44.

[27] Pearl, J. Probabilistic reasoning in intelligent systems: networks of plausible inference. Morgan Kaufmann Publishers Inc., 1988.

[28] Pearl, J. Causality: Models, Reasoning, and Inference. Cambridge University Press, 2000.

[29] Pearl, J. Reasoning with Cause and Effect. AI Magazine 23, 1 (2002), $95-112$.

[30] Perrin, T., Kawai, H., Kunieda, K., and Yamada, K. Global Dynamics Network Construction from the Web. In Information-Explosion and Next Generation Search, 2008. INGS'08. International Workshop on (2008), pp. 69-76.

[31] Picard, R. Affective Computing. MIT Press, 1997.

[32] Pinker, S. The Stuff of Thought: Language as a Window into Human Nature. Penguin Books, 2007. 
[33] Pohl, J. Some Notions of Complex Adaptive Systems and their Relationship to Our World. In Advances in Computer-Based and Web-Based Collaborative Systems, focus symposium: International Conference on Systems Research, Informatics and Cybernetics (InterSymp-99), Baden-Baden, Germany (1999), pp. 9-24.

[34] Price, H. Agency and Causal Asymmetry. Mind 101, 403 (1992), 501-520.

[35] Sah, R., And Stiglitz, J. Human Fallibility and Economic Organization. The American Economic Review 75, 2 (1985), 292-297.

[36] Sah, R., and Stiglitz, J. The Architecture of Economic Systems: Hierarchies and Polyarchies. The American Economic Review 76, 4 (1986), $716-727$.

[37] Saito, H., Kawai, H., Tsuchida, M., Mizuguchi, H., and Kusui, D. Extraction of Statistical Terms and Co-occurrence Networks from Newspapers. In Proceedings of NTCIR-6 Workship Meeting, May 15-18, Tokyo (2007), pp. 256-263.

[38] Sakai, H., And Masuyama, S. Cause Information Extraction from Financial Articles Concerning Business Performance. IEICE Transactions on Information and Systems E91-D, 4 (2008), 959-968.

[39] Sanchez-Graillet, O., And Poesio, M. Acquiring Bayesian Networks from Text. In Proc. of the 4 th International Conference on Language Resources and Evaluation (LREC-2004) (2004), pp. 955-958.

[40] Segaran, T. Programming Collective Intelligence: Building Smart Web 2.0 Applications. O'Reilly Media, Inc., 2007. 
[41] Sheffrin, S., And Triest, R. A New Approach to Causality and Economic Growth. Federal Reserve Bank of Boston, 1995.

[42] Simon, H. On the Definition of the Causal Relation. The Journal of Philosophy 49, 16 (1952), 517-528.

[43] Simon, H. Causal Ordering and Identifiability, vol. 11. New York: John Wiley \& Sons, 1953, ch. III, pp. 49-74.

[44] Simon, H. Spurious Correlation: A Causal Interpretation. Journal of the American Statistical Association 49, 267 (1954), 467-479.

[45] Simon, H. Rationality as Process and as Product of Thought. American Economic Review 68, 2 (1978), 1-16.

[46] Simon, H. The Sciences of the Artificial. MIT Press, 1996.

[47] ter Meulen, A. Representing Time in Natural Language: The Dynamic Interpretation of Tense and Aspect. MIT Press, 1995.

[48] Weiss, S., Kulikowski, C., And Safir, A. A Model-Based Consultation System for the Long-Term Management of Glaucoma. In Proceedings of IJCAI (1977), vol. 77, pp. 826-831.

[49] Wu, D., And Butz, C. On the Complexity of Probabilistic Inference in Singly Connected Bayesian Networks. Lecture Notes in Computer Science 3641 (2005), 581-590.

[50] Wu, F., Yuan, X., Huang, W., and Chen, Y. Heterosubtypic Protection Conferred by Combined Vaccination with M2e Peptide and Split Influenza Vaccine. Vaccine (2008). 
[51] Zadeh, L. Causality Is Undefinable. In Abstract Of A Lecture Presented At The BISC Seminar, University of California, Berkeley (2001).

[52] ZADEH, L. Causality is Undefinable-Toward a Theory of Hierarchical Definability. In The 10th IEEE International Conference on Fuzzy Systems (2001), IEEE, pp. 67-68. 